\title{
Preface to the MME Special Issue on Critical Minerals Part I
}

\section{Virginia T. McLemore ${ }^{1}$}

Published online: 21 August 2019

(C) Society for Mining, Metallurgy \& Exploration Inc. 2019

Minerals are essential to economic development and maintaining our way of life, and are required in a wide variety of products within the civilian, energy, and military sectors of the economy. Every year, the Minerals Education Coalition of the Society for Mining, Metallurgy \& Exploration (SME) updates its Mineral Baby poster (https:// mineralseducationcoalition.org/mining-mineral-statistics) - in 2019, every baby born in the USA will require 3.19 million pounds of minerals, metals, and fuels in their lifetime. Many of these commodities are critical minerals, and access to these minerals is vital to continuing life the way we know it. As modern society becomes more dependent upon the numerous commodities and products required to maintain our standard of living, critical minerals will become increasingly important in the future. This special issue is devoted to critical minerals.

In industry, the term minerals refers to any rock, mineral, or other naturally occurring materials of economic value, including metals, industrial minerals, energy minerals, gemstones, and aggregates. Although there are many definitions of critical minerals that depend upon many factors, especially the degree to which a mineral is essential, risk of potential disruption in supply, and national strategic requirements [1], the definition proposed by U.S. President Donald Trump will be used here. In December 2017, President Trump signed an executive order (Presidential Executive Order (EO) No. 13,817) [2] that required the Departments of Interior and Defense to develop a list of critical minerals. In May 2018, the U.S. Department of the Interior published its final list of 35 critical minerals

Virginia T. McLemore

virginia.mclemore@nmt.edu

1 New Mexico Bureau of Geology and Mineral Resources, New Mexico Institute of Mining and Technology, Socorro, NM, USA
(Table 1). As defined by EO No. 13,817, “a critical mineral is a mineral (1) identified to be a nonfuel mineral or mineral material essential to the economic and national security of the United States, (2) from a supply chain that is vulnerable to disruption, and (3) that serves an essential function in the manufacturing of a product, the absence of which would have substantial consequences for the U.S. economy or national security." The concept of critical and strategic minerals is not new. It emerged in the USA after World War 1 primarily to meet future defense needs [3]. Most critical minerals are $100 \%$ imported into the USA [4]. However, specific critical minerals may vary between different countries (Table 1) [1, 5-8]. In many cases, geological resources are available in the world, but the real issue is risk of potential supply disruptions. Disruptions in supply chains can arise for any number of reasons, including natural disasters, labor strife, trade disputes, resource nationalism, conflict, and civil war. Therefore, understanding how critical minerals fit into the mine life cycle becomes important, along with research in mineral policy, permitting, exploration, geologic processes in forming the deposits, mineral processing, reprocessing of mine wastes, recycling, and environmental issues. The list of critical minerals changes with time and changing of society's needs within specific countries. What was considered a critical mineral in the past may not be considered as such today: For example, table salt $(\mathrm{HCl})$ was once considered a critical mineral by the Romans and the Latin phrase "salarium argentum," or "salt money," (that is, salary) referred to part of the payment made to Roman soldiers. Today, table salt is abundant, no longer considered a critical mineral, and produced as a primary product or a by-product from several types of deposits.

This is the first of two issues of Mining, Metallurgy \& Exploration (MME) that focus on critical minerals. In these issues, papers spanning the mine life cycle, including mineral policy, exploration, geology, mineralogy, mineral processing, disposal of mine wastes, reprocessing of 
Table 1 Critical minerals in the USA and their primary industry uses $[1,2]$

\begin{tabular}{|c|c|c|c|c|c|c|c|c|}
\hline Mineral & Aerospace & Defense & Energy & $\begin{array}{l}\text { Telecommunications } \\
\text { and electronics }\end{array}$ & Transportation & Other & $\begin{array}{l}\text { Top-producing } \\
\text { country }\end{array}$ & $\begin{array}{l}\text { Top country } \\
\text { supplier }\end{array}$ \\
\hline Aluminum (bauxite) & $\mathrm{x}$ & $\mathrm{x}$ & $\mathrm{x}$ & $\mathrm{x}$ & $\mathrm{x}$ & $\mathrm{x}$ & China & Canada \\
\hline Antimony & & $\mathrm{x}$ & $\mathrm{x}$ & $\mathrm{x}$ & & $\mathrm{x}$ & China & China \\
\hline Arsenic & & $\mathrm{x}$ & $\mathrm{x}$ & $\mathrm{x}$ & & $\mathrm{x}$ & China & China \\
\hline Barite & & & $\mathrm{x}$ & $\mathrm{x}$ & & $\mathrm{x}$ & China & China \\
\hline Beryllium & $\mathrm{x}$ & $\mathrm{x}$ & $\mathrm{x}$ & $\mathrm{x}$ & & $\mathrm{x}$ & U.S. & Kazakhstan \\
\hline Bismuth & & $\mathrm{x}$ & $\mathrm{x}$ & $\mathrm{x}$ & & $\mathrm{x}$ & China & China \\
\hline Cesium & $\mathrm{x}$ & $\mathrm{x}$ & $\mathrm{x}$ & $\mathrm{x}$ & & $\mathrm{x}$ & Canada & Canada \\
\hline Chromium & $\mathrm{x}$ & $\mathrm{x}$ & $\mathrm{x}$ & $\mathrm{x}$ & $\mathrm{x}$ & $\mathrm{x}$ & South Africa & South Africa \\
\hline Cobalt & $\mathrm{x}$ & $\mathrm{x}$ & $\mathrm{x}$ & $\mathrm{x}$ & $\mathrm{x}$ & $\mathrm{x}$ & Congo & Norway \\
\hline Fluorspar & & & $\mathrm{x}$ & $\mathrm{x}$ & & $\mathrm{x}$ & China & Mexico \\
\hline Gallium & $\mathrm{x}$ & $\mathrm{x}$ & $\mathrm{x}$ & $\mathrm{x}$ & & $x$ & China & China \\
\hline Germanium & $\mathrm{x}$ & $\mathrm{x}$ & $\mathrm{x}$ & $\mathrm{x}$ & & $x$ & China & China \\
\hline Graphite (natural) & $\mathrm{x}$ & $\mathrm{x}$ & $\mathrm{x}$ & $\mathrm{x}$ & $\mathrm{x}$ & $\mathrm{x}$ & China & China \\
\hline Hafnium & $\mathrm{x}$ & $\mathrm{x}$ & $\mathrm{x}$ & $\mathrm{x}$ & & $\mathrm{x}$ & Australia & China \\
\hline Helium & & & & $\mathrm{x}$ & & $\mathrm{x}$ & U.S. & Qatar \\
\hline Indium & $\mathrm{x}$ & $\mathrm{x}$ & $\mathrm{x}$ & $\mathrm{x}$ & & $\mathrm{x}$ & China & Canada \\
\hline Lithium & $\mathrm{x}$ & $\mathrm{x}$ & $\mathrm{x}$ & $\mathrm{x}$ & $\mathrm{x}$ & $\mathrm{x}$ & Australia & Chile \\
\hline Magnesium & $\mathrm{x}$ & $\mathrm{x}$ & $\mathrm{x}$ & $\mathrm{x}$ & $\mathrm{x}$ & $\mathrm{x}$ & China & China \\
\hline Manganese & $\mathrm{x}$ & $\mathrm{x}$ & $\mathrm{x}$ & $\mathrm{x}$ & $\mathrm{x}$ & $\mathrm{x}$ & China & South Africa \\
\hline Niobium & $\mathrm{x}$ & $\mathrm{x}$ & $\mathrm{x}$ & $\mathrm{x}$ & & $\mathrm{x}$ & Brazil & Brazil \\
\hline Platinum Group Metals (PGM)* & $\mathrm{x}$ & & $\mathrm{x}$ & $\mathrm{x}$ & $\mathrm{x}$ & $\mathrm{x}$ & South Africa & South Africa \\
\hline Potash & & & $\mathrm{x}$ & $\mathrm{x}$ & & $\mathrm{x}$ & Canada & Canada \\
\hline Rare Earth Elements** & $\mathrm{x}$ & $\mathrm{x}$ & $\mathrm{x}$ & $\mathrm{x}$ & $\mathrm{x}$ & $\mathrm{x}$ & China & China \\
\hline Rhenium & $\mathrm{x}$ & & $\mathrm{x}$ & $\mathrm{x}$ & & $\mathrm{x}$ & Chile & Chile \\
\hline Rubidium & $\mathrm{x}$ & $\mathrm{x}$ & $\mathrm{x}$ & $\mathrm{x}$ & & $\mathrm{x}$ & Canada & Canada \\
\hline Scandium & $\mathrm{x}$ & $\mathrm{x}$ & $\mathrm{x}$ & $\mathrm{x}$ & & $\mathrm{x}$ & China & China \\
\hline Strontium & $\mathrm{x}$ & $\mathrm{x}$ & $\mathrm{x}$ & $\mathrm{x}$ & $\mathrm{x}$ & $\mathrm{x}$ & Spain & Mexico \\
\hline Tantalum & $\mathrm{x}$ & $\mathrm{x}$ & $\mathrm{x}$ & $\mathrm{x}$ & & $\mathrm{x}$ & Rwanda & China \\
\hline Tellurium & & $\mathrm{x}$ & $\mathrm{x}$ & $\mathrm{x}$ & & $\mathrm{x}$ & China & Canada \\
\hline Tin & & $\mathrm{x}$ & & $\mathrm{x}$ & & $\mathrm{x}$ & China & Peru \\
\hline Titanium & $\mathrm{x}$ & $\mathrm{x}$ & $\mathrm{x}$ & $\mathrm{x}$ & & $\mathrm{x}$ & China & South Africa \\
\hline Tungsten & $\mathrm{x}$ & $\mathrm{x}$ & $\mathrm{x}$ & $\mathrm{x}$ & & $\mathrm{x}$ & China & China \\
\hline Uranium & $\mathrm{x}$ & $\mathrm{x}$ & $\mathrm{x}$ & & & $\mathrm{x}$ & Kazakhstan & Canada \\
\hline Vanadium & $\mathrm{x}$ & $\mathrm{x}$ & $\mathrm{x}$ & $\mathrm{x}$ & & $\mathrm{x}$ & China & South Africa \\
\hline Zirconium & $\mathrm{x}$ & $\mathrm{x}$ & $\mathrm{x}$ & $\mathrm{x}$ & & $\mathrm{x}$ & Australia & China \\
\hline
\end{tabular}

*Platinum group metals include ruthenium, rhodium, palladium, osmium, iridium, and platinum

**Rare earth elements include the 15 lanthanide elements (lanthanum, cerium, praseodymium, neodymium, promethium, samarium, europium, gadolinium, terbium, dysprosium, holmium, erbium, thulium, ytterbium, and lutetium), and yttrium and scandium are often also included as rare earth elements

mine wastes, and environmental issues are covered. These papers not only provide insights into a wide variety of critical minerals but also highlight some of the future research needs to ensure that we have sustainable supplies of these critical minerals. This special issue should be of value to the exploration and mining industries, public policy and decision makers, and others interested in the evolving issue of critical minerals.

Virginia T. McLemore, Ph.D., CPG

Section Editor-in-Chief, Exploration

Mining, Metallurgy \& Exploration (MME) 


\section{References}

1. Jowitt SM, Mudd GM, Werner TT, Weng Z, Barkoff DW, McCaffrey D (2018) The critical metals: an overview and opportunities and concerns for the future. In: Arribas AM, Mauk JL (eds) Metals, minerals, and society: economic geology, Special Publication no. 21, pp 25-38

2. U.S. Executive Order No. 13,817, 2017, Presidential executive order on a federal strategy to ensure secure and reliable supplies of critical minerals: https:/www.whitehouse.gov/presidential-actions/ presidential-executiveorder-federal-strategy-ensure-secure-reliablesupplies-critical-minerals/

3. National Research Council (2008) Managing materials for a twentyfirst century military. The National Academies Press, 207 p, Washington, D.C. https://doi.org/10.17226/12028

4. U.S. Geological Survey (2019) Mineral commodity summaries 2019: U.S. Geological Survey, 200 p. https://doi.org/10.3133/ 70202434

5. Committee on Critical Mineral Impacts of the U.S. Economy, 2008, Minerals, critical minerals, and the U.S. Economy: Committee on
Earth Resources, National Research Council, ISBN: 0-309-112834, 264 p., http://www.nap.edu/catalog/12034.html

6. Subcommittee on Critical and Strategic Mineral Supply Chains Committee on Environment, Natural Resources, and Sustainability (2018) Assessment of critical minerals: updated application of screening methodology: National Science and Technology Council, 7 p., https://www.whitehouse.gov/wp-content/uploads/2018/02/ Assessment-of-Critical-Minerals-Update-2018.pdf

7. National Science and Technology Council (NSTC) (2016) Assessment of critical minerals: screening methodology and initial application. Executive office of the President, Washington, D.C., 57 p., https:/www.whitehouse.gov/sites/whitehouse.gov/files/images/ CSMSC\%20Assessment\%20of\%20Critical\%20Minerals\% 20Report\%202016-03-16\%20FINAL.pdf

8. Hofmann M, Hofmann H, Hagelüken C, Hool A (2018) Critical raw materials: a perspective from the materials science community: sustainable materials and technologies. 17:1-10

Publisher's Note Springer Nature remains neutral with regard to jurisdictional claims in published maps and institutional affiliations. 